\title{
Internet da Coisas (IoT): Definições e aplicabilidade aos usuários finais
} IInternet of Things (IoT): Definitions
and applicability for end users

Patrícia Carrion ${ }^{1}$

Manuela Quaresma² 


\section{Resumo}

A interconexão de dispositivos físicos com capacidades computacionais de detecção e comunicação de dados não é uma concepção nova, porém a compreensão da real aplicabilidade de uma Internet das Coisas (Internet of Things, ou loT) ainda é incipiente. A Internet das Coisas descreve o que se observa hoje com o crescente número de dispositivos conectados habilitados à internet. Isto é, um estado onde "coisas", de pequenos objetos a ambientes e cidades, veem-se capacitados a se comunicar uns com os outros, e consequentemente com os humanos presentes no ecossistema. Neste artigo, buscou-se discutir como a realidade de produtos cada vez mais conectados vem transformando o modo como usuários e organizações interagem com tecnologias em níveis novos e distintos. Para tanto, foram traçadas definições acerca da loT, de suas aplicações voltadas ao usuário final, e de como os serviços loT, centradas no cliente-usuário, dependem de fatores instituídos ainda no processo de design.

Palavras-chave: Internet das Coisas, Interação Humano-Computador, Dispositivos conectados.

\section{Abstract}

The interconnection of physical devices with computational capabilities of data detection and communication is not a new concept, but the understanding of an Internet of Things (IOT) ecosystem's real applicability is still insipid. The Internet of Things describes what we see today with the growing number of connected devices enabled to the internet. That is, a state where "things", from small objects to environments and cities, are able to communicate with one another, and consequently with the humans present in the ecosystem. In this article, we sought to discuss how the reality of increasingly connected products has transformed the way users and organizations interact with technologies at new and different levels. To that end, we draw definitions about loT, its end-user applications, and how loT services, centered on the user-client, depend on factors that must be instituted during the design process.

Key-words: Internet of Things, Human-Computer Interaction, Connected devices.

ISSN: 2316-7963

${ }^{1}$ M. Sc, PUC - Rio (patriciatpc@gmail.com)

${ }^{2}$ D. Sc, PUC - Rio (mquaresma@puc-rio.br) 


\title{
1 Introdução
}

Desde os primeiros computadores de uso pessoal a partir de 1980, a experiência e a interação de usuários com esses dispositivos - e posteriormente também com a conexão via internet - ocorreram de modo estático, com pouca ou nenhuma mobilidade (CAMPBELL-KELLY et al., 2014). Com a evolução tecnológica nas décadas finais do século XX, em decorrência de uma maior capacidade de processamento dos chips e da microinformática, os computadores se tornaram cada vez menores e mais portáteis (CASTELLS, 2011). Por consequência, no cenário atual e desde 2014, no Brasil (IBGE, 2014) e nos EUA (LELLA e LIPSMAN, 2014) o uso da internet móvel superou o uso da mesma em computadores desktop. É possível inferir, portanto, que uma quantidade significativa de pessoas carrega pelo menos um dispositivo conectado à internet o tempo todo, sendo o acesso a serviços e conteúdos on-line uma parte intrínseca do cotidiano.

Hoje, o uso de dispositivos com capacidades computacionais e acesso à internet é algo que observamos e experimentamos principalmente através de telas (ROWLAND et al., 2015). Porém, iniciativas e previsões de um mundo de objetos conectados para além da tela de um computador existem desde antes da primeira geração de computadores na década de 1940 (COMPUTER HISTORY MUSEUM, 2016). Em entrevista à revista Colliers em 1926, o inventor, engenheiro, físico e futurista Nikola Tesla previu a conexão de dispositivos e pessoas via tecnologia sem fio:

\begin{abstract}
Quando a rede sem fio for perfeitamente aplicada, a Terra inteira será convertida em um cérebro enorme [...]. Nós deveremos poder nos comunicar instantaneamente, independentemente da distância. Não só isso, mas através da televisão e da telefonia, veremos e nos ouviremos tão perfeitamente como se estivéssemos cara a cara, apesar das distâncias intervenientes de milhares de quilômetros; e os instrumentos através dos quais poderemos fazer isso serão incrivelmente simples em comparação ao nosso telefone atual. Um homem poderá carregá-los no bolso do colete. (TESLA, 1926:2013, tradução nossa).
\end{abstract}

Décadas depois, em 1964, McLuhan (1964:1994) complementou essa noção de objetos conectados ao afirmar que através de meios elétricos, criar-se-ia uma dinâmica pela qual todas as tecnologias - incluindo as cidades - seriam traduzidas em sistemas de informação. Quase três décadas mais tarde, em 1990, um ano após a idealização da World Wide Web - um conceito de aplicação de documentos hipermídia bilateral, em que havia o lado do servidor e o lado do usuário (CAMPBELL-KELLY et al., 2014) - o cientista da computação e inventor John Romkey criou uma torradeira que poderia ser ligada e desligada pela internet. Ela é considerada o primeiro de muitos dispositivos de um ecossistema que viria a ser chamado de loT (Internet of Things, ou Internet das Coisas). Sobre a própria inovação, Romkey (2017, tradução nossa) explica que "anteriormente, o mundo tinha uma internet, mas era uma internet sem "coisas".

1 MIT, de Massachusetts Institute of Technology, ou Instituto de Tecnologia de Massachusetts.

20 método de RFID (Radio-Frequency Identification, ou Identificação por Radiofrequência) usa campos eletromagnéticos para reconhecer e rastrear automaticamente etiquetas anexadas a objetos. Essas etiquetas contêm informações armazenadas eletronicamente. (WEIS, 2007) 
Não havia interruptores de luz inteligentes ou termostatos, nem Fitbits ou câmeras Wi-Fi. Nós não estávamos tão conectados, e certamente não éramos quantificados".

Por fim, em 1999, o termo Internet das Coisas (IoT) foi cunhado pelo pesquisador do MIT Kevin Ashton, durante uma apresentação de negócios feita à Procter \& Gamble (P\&G). Nesse cenário, Ashton sugerira a ideia de utilizar etiquetas RFID na cadeia de suprimentos da empresa (ASHTON, 2011). De acordo com o pesquisador, seres humanos, assim como o ambiente que os rodeia, são físicos, e a economia e a sociedade são baseadas em coisas. "No entanto, a tecnologia da informação de hoje é tão dependente dos dados originados pelas pessoas que nossos computadores sabem mais sobre ideias do que coisas" (ASHTON, 2011). Em sincronia com Ashton, Gershenfeld (1999, tradução nossa) mencionou, no mesmo ano, a importância das "coisas" na evolução do panorama tecnológico, ao explicar que o crescimento rápido da World Wide Web foi apenas "a carga de gatilho que agora está desencadeando a explosão real, já que as coisas começam a usar a Net".

Uma vez que a Internet das Coisas se tornou conhecida, o conceito que a permeia passou a ser referido por meio de diversos outros termos, como internet física, computação ubíqua, comunicação M2M (Machine-to-Machine, ou Máquina-a-Máquina), Web of Things (ou Rede das Coisas), ambientes conectados, cidades inteligentes, redes de sensores sem fio, entre outros. Porém, o que é, de fato, e quais são as "coisas" conectadas no universo loT? Além disso, quais são as possibilidades de aplicações de loT que beneficiam hoje e no futuro o dia a dia de usuários e organizações?

\section{0 que é a Internet das Coisas?}

Em 1950, o cientista e matemático Alan Turing defendeu a possibilidade da existência de uma inteligência artificial. Para Turing (1950), as máquinas poderiam competir com os seres humanos em todos os campos puramente intelectuais, e, para tanto, o melhor seria “[...] fornecer à máquina os melhores órgãos sensoriais que o dinheiro pode comprar e depois ensiná-la a entender e a falar em inglês. Esse processo poderia seguir o ensino normal de uma criança". A previsão de Turing converge com a ideia de uma realidade em que coisas seriam capacitadas com identidades e "personalidades virtuais". Para Bassi e Horn (2008) tais coisas são as que operam em espaços inteligentes, e usam interfaces inteligentes, para se conectarem e se comunicarem dentro de contextos sociais, ambientais e de usuários.

De acordo com os autores, as coisas seriam objetos interconectados, com papel ativo no que pode ser chamado de internet futura. Em termos concretos, a origem semântica da expressão Internet das Coisas é composta por duas palavras e conceitos: em "Internet", tem-se o protocolo de comunicação, e em "Coisas", objetos não identificáveis com precisão. À vista disso, semanticamente, "Internet das Coisas" significa uma rede mundial de objetos interligados, com base em protocolos de comunicação (BASSI e HORN, 2008). Em definição mais abstrata, Easterling (2012, tradução nossa), vislumbra que a loT descreve "um mundo embutido com tantos dispositivos digitais que o espaço entre eles não consiste em circuitos obscuros, mas sim no espaço da 
própria cidade". Para o autor, o computador extrapolou os limites físicos de sua estrutura, fazendo com que os objetos comuns do cotidiano assumissem sinais digitais.

Em suma, a Internet das Coisas trata-se de um ecossistema que conecta objetos físicos, através de um endereço de IP ${ }^{3}$ ou outra rede, para trocar, armazenar e coletar dados para consumidores e empresas através de uma aplicação de software. $O$ fluxo de dados nesse contexto pode ser entendido como a lista a seguir, que aponta a transformação dos dados de um objeto inteligente para os consumidores finais (MOON, 2016):

- Sensores em máquinas - Primeiro, sensores "sentem" o entorno do ambiente e coletam dados. Ex.: Smartphones, roteadores, beacons ${ }^{4}$, wearables (dispositivos vestíveis), termômetros e afins;

- Centro de dados (Cloud) - Segundo, os dados transportados a partir das máquinas conectadas são armazenados e analisados por meio de computação em nuvem;

- Aplicação (Software) - Depois, aplicações controlam os dados analisados e fornecem serviços ao usuário final;

- Consumidor - Por fim, o consumidor (usuário final) compartilha informações úteis com serviços e outras pessoas.

Moon (2016) explica haver distinções no meio loT no que se refere a serviços e produtos voltados às aplicações na indústria e aos consumidores, ou seja, usuários finais. Entende-se então a ocorrência de uma Industrial IoT (IloT, ou "Internet das Coisas Industriais") em contraponto a uma Consumer loT (CloT, ou "Internet do Consumidor de Coisas"). Para o autor, as inovações no campo do consumo tratam-se principalmente de produtos de hardware, enquanto as de cunho industrial tem sido principalmente de software (ou seja, no âmbito da inteligência artificial, da aprendizagem de máquina e demais métodos de análise de dados). Rowland et al. (2015) acrescentam que o foco da Consumer loT está em novas e imersivas experiências - centradas no cliente-usuário - que dependem de fatores instituídos ainda no processo de design dos serviços e produtos. Nesse sentido, a complexidade do design da experiência do usuário para loT depende de alguns fatores, entre eles:

- Do nível de maturidade da tecnologia com a qual se está trabalhando;

- Do contexto de uso e/ou das expectativas que os usuários têm com o sistema;

- Da complexidade do serviço (por exemplo, quantos dispositivos o usuário tem para interagir).

Exemplos desses fatores e de aplicações de Internet das Coisas serão abordadas no decorrer deste artigo.

3 IP (Internet Protocol, ou Protocolo de Internet) é um rótulo numérico atribuído a cada dispositivo conectado a uma rede de computadores. Cada dispositivo com capacidade computacional possui um IP único.

4 Beacons são pequenos dispositivo sem fio que transmitem sinal de rádio contínuo. 0 sinal é detectado por dispositivos inteligentes próximos via Bluetooth SmartTM, tecnologia compatível com smartphones, computadores desktop e laptops, e consoles de videogame. (GASIOREK, 2014) 


\section{IoT: Uma realidade em expansão}

A Internet das Coisas (IoT) descreve a revolução já em curso que pode ser observada no número crescente de dispositivos habilitados para internet. Nesse contexto, a loT refere-se a um estado onde "coisas", como objetos, ambientes, veículos e roupas estão capacitados a terão cada vez mais informações associadas a eles, e podem se conectar e se comunicar uns com os outros e com demais dispositivos habilitados para a web (REVELL, 2013).

A expectativa é a de que a Internet das Coisas irá infundir cerca de 7 trilhões de dólares para a economia mundial até 2020 , uma tendência prevista há anos que finalmente está se concretizando agora (CompTIA, 2015). Em relatório produzido pela CompTIA (2015), uma organização sem fins lucrativos, o número de coisas conectadas, desde computadores, perpassando monitores domésticos até carros, deverá crescer a uma taxa composta anual de 23,1\% entre 2014 e 2020, chegando a 50 biIhões de coisas no ano em questão. O relatório, que teve como base os resultados de duas pesquisas realizadas com cerca de 900 pessoas - entre profissionais da indústria de TI e clientes -, captou ainda quais seriam os benefícios esperados da loT, e como estes se relacionam com as atividades e operações já existentes. De acordo com os entrevistados, os cinco principais benefícios esperados são: a economia de custos de eficiência operacional; novos e melhores fluxos de dados para melhorar a tomada de decisões; ganhos de produtividade; melhor visibilidade e monitoramento de pessoas, serviços e produtos; e novas e melhores experiências dos clientes.

Esses dados se assemelham aos captados pela Ericsson que, de acordo com seu relatório de mobilidade (HEUVELDOP, 2017), prevê a existência de mais de 30 bilhões de dispositivos conectados - dos quais cerca de 20 bilhões estarão relacionados ao ecossistema de loT -até 2023. Entre os dispositivos loT têm-se carros, máquinas, medidores, sensores, terminais de ponto de venda, eletrônicos de consumo e wearables. Entre 2017 e 2023, os dispositivos loT conectados deverão aumentar a uma taxa de crescimento anual composta de $19 \%$.

Por fim, pesquisa divulgada pelo IHS Markit (LUCERO, 2016) mostra o número de dispositivos conectados em todo o mundo de 2015 a 2025 (Gráfico 1). Para 2020, a base instalada de dispositivos da Internet de Coisas deverá chegar a pouco mais de 30 bilhões em todo o mundo, e a expectativa é a de que o mercado global de loT valha mais de um bilhão de dólares americanos anualmente a partir de 2017. 


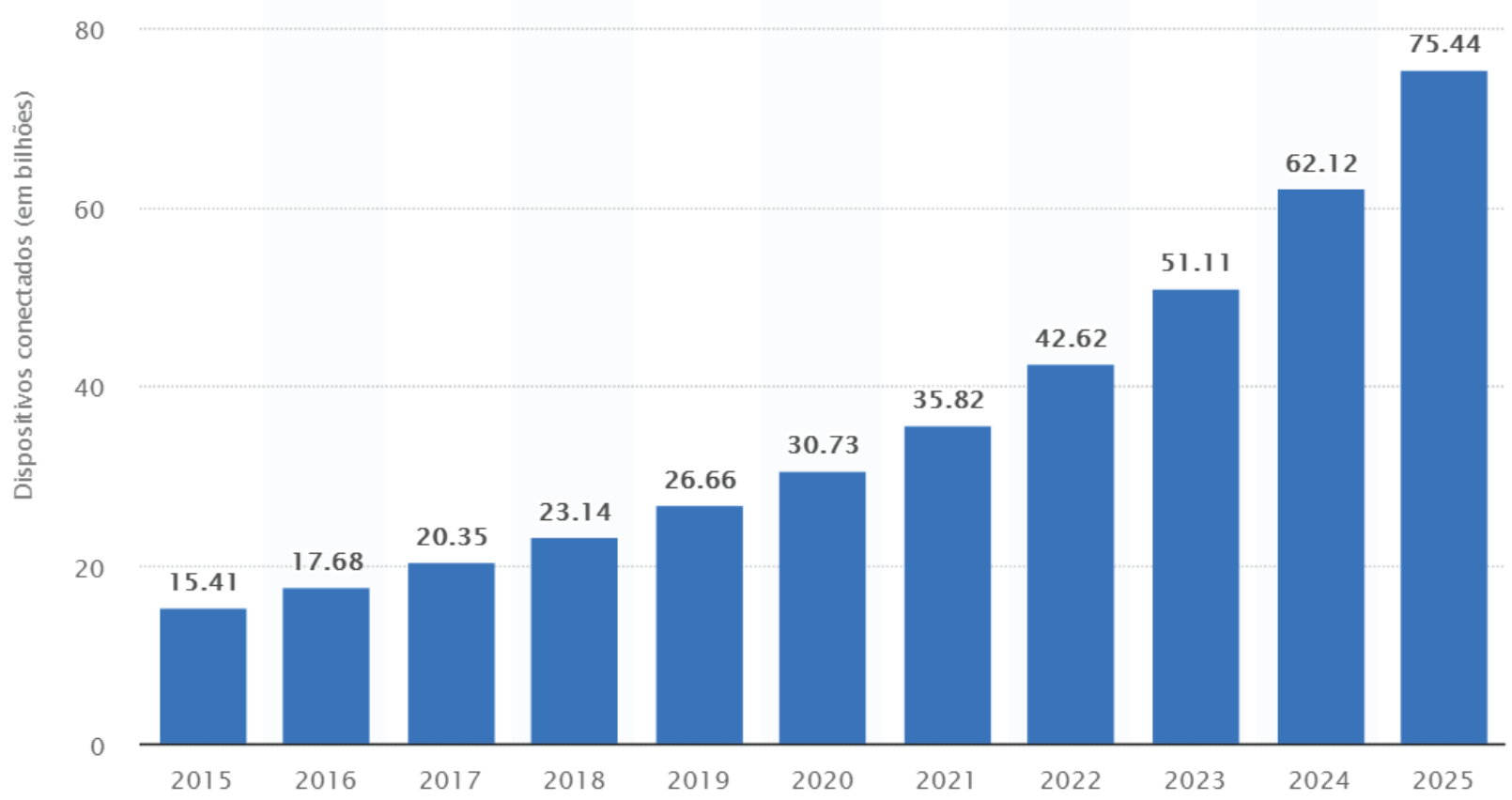

Gráfico 1 - Dispositivos conectados de Internet das Coisas instalados em todo o mundo de 2015 a 2025 (em bilhões). Fonte: adaptado de IHS Markit (2016)

A Internet das Coisas é um termo abrangente para uma ampla gama de tecnologias e serviços subjacentes que, por sua vez, fazem parte de um ecossistema mais amplo. A interconexão de dispositivos físicos com possibilidades de detecção e comunicação (por meio de sensores e atuadores), não é um conceito novo, no entanto, no que cerne à Internet das Coisas, os endpoints físicos estão conectados através de endereços de IP exclusivos pelos quais dados podem ser reunidos e comunicados. Com previamente elucidado, essa comunicação de dados acontece através de software, sendo, por fim, armazenada na nuvem.

Por si só a Internet das Coisas se trata de objetos inteligentes com capacidades sensoriais, mas é somente através de aplicações de consumo que ela se torna uma "Internet das Pessoas" (IoP) (MIRANDA et al., 2015). E é nesse encontro de Coisas e Pessoas que se tem a maioria das aplicações, serviços e produtos de loT para consumo. Conforme Miranda et al. (2015), o objetivo principal no desenvolvimento de aplicações para a loT está na integração da tecnologia a soluções benéficas aos usuários. Todavia, ainda que o sucesso e o consequente crescimento da loT sejam inquestionáveis, "[...] a interação das pessoas com esse tipo de sistema ainda está longe de ser amigável" (MIRANDA et al., 2015, tradução nossa). De acordo com os autores, as formas como as tecnologias no campo da loT estão atualmente integradas aos usuários não estão preparadas a se adequarem aos contextos de usos. Por isso, ao invés de "funcionar para as pessoas", as tecnologias tendem a forçar os usuários a se adaptarem aos requisitos tecnológicos, ou mesmo a serem conscientes do sistema.

Partindo de uma perspectiva de Internet das Pessoas, pode-se inferir que, no mercado da loT, somente dispositivos que se conectam profundamente com seus 
usuários terão poder de permanência (HORAN, 2016). Nos últimos anos, a adoção acelerada de tecnologias como telas sensíveis ao toque, computação em nuvem, realidades virtual e aumentada, interfaces gestuais, sensores, entre outros, transformaram o desenvolvimento de produtos. Porém, para Kuniavsky (2016, tradução nossa), a transição do mundo para uma esfera em que a capacidade computacional se faz onipresente resulta em muitas ideias equivocadas quanto ao que é e para quem é a Internet das Coisas -"simplesmente conectar à internet coisas já existentes não produz valor ao cliente".

Kuniavsky (2016) explica que a conectividade pura e simples auxilia quando se espera maximizar a eficiência de um processo fixo, o que, no entanto, não é um problema que a maioria das pessoas enfrenta. Isto, porque, seres humanos são capazes de se conectar com vários dispositivos a um computador há algum tempo. O problema, na verdade, está na qualidade da experiência do usuário, que surge no momento em que este precisa se conectar a diversos pontos por meio de uma variedade de dispositivos, interpretando os significados de todos esses sensores a fim de criar um valor personalizado.

Rowland et al. (2015) também apontam desafios de design de experiência na Internet das Coisas, sendo estes relacionados: aos múltiplos dispositivos com capacidades distintas; aos diferentes tipos de tecnologias acopladas; à expectativa e tolerância dos usuários diante de falhas; e ao caráter assíncrono da loT. Uma forma de exemplificar a experiência de usuários finais nesse ecossistema está em entender como o conceito de Internet das Coisas vem sendo abordado pelo mercado. Além disso, é válido destacar também como iniciativas da IloT, Internet das Coisas Industriais, prometem mudar drasticamente a forma como empresas do Fortune 500 continuam operando. Nesse sentido, a IloT tem o potencial de ser ainda maior do que loT para consumidores (MOON, 2016).

\section{Aplicações e interfaces de IoT}

À medida que os produtos se tornam cada vez mais conectados, eles se comunicam e interagem com usuários e organizações em níveis novos e distintos. Dispositivos loT estão alterando esferas diversas da sociedade, desde o gerenciamento doméstico e os cuidados com a saúde, à implementação nas indústrias, no planejamento urbano e de transportes, e na geração de energia, por exemplo. No quadro a seguir (Quadro 1), têm-se as aplicações possíveis de loT já em andamento hoje, e algumas destas serão abordadas em detalhes em seguida. Para fins organizacionais, elas estão apresentadas de forma seccionada entre aplicações macro, geralmente presentes no âmbito da indústria, e micro, no de produtos e serviços voltados - e mais evidentes - aos usuários finais.

5 Endpoints são dispositivos de usuário único, com interface "humana" (ou "para humanos") e que possuem endereço de IP (KAY, 2014).

6 O Fortune Global 500, também conhecido como Global 500, é um ranking anual das 500 maiores empresas do mundo, medido conforme a receita das mesmas. A lista é compilada e publicada anualmente pela revista Fortune. Fonte: Fortune - Global 500 < http://fortune.com/global500/>. Acesso em: 21 dez. 2017. 


\begin{tabular}{|c|c|}
\hline Aplicações Macro (Indústria) & Aplicações Micro (Consumo) \\
\hline \multicolumn{2}{|l|}{ Indústria* } \\
\hline Cidades Inteligentes (Smart Cities) & Dispositivos vestíveis (Wearables) \\
\hline Energia & Casas Inteligentes (Smart Homes) \\
\hline Transportes & Carros \\
\hline Agricultura e Pecuária & Varejo \\
\hline \multicolumn{2}{|l|}{ Saúde ${ }^{* *}$} \\
\hline \multicolumn{2}{|c|}{$\begin{array}{l}\text { * Em específico no campo da manufaturação e de recursos naturais, como óleoe gás. } \\
\text { ** Aplicações na saúde também existem no meio de wearables, mas aqui referem-se às voltadas ao gerenciamento } \\
\text { hospitalar, por exemplo. }\end{array}$} \\
\hline
\end{tabular}

Quadro 1 - Aplicações macro e micro de loT hoje, nos âmbitos da lloT e da CloT. Fonte: Elaboração da autora, 2017.

Fonte: 0 autor

\subsection{Aplicações: cidades inteligentes, transportes e varejo}

A população humana nas cidades está crescendo exponencialmente devido à migração maciça e à urbanização, e a expectativa é a de que a população mundial vivendo em áreas urbanas deverá aumentar de 54\% em 2014 para 66\% até 2050 (UNITED NATIONS, 2014). Nesse panorama, tem-se a preocupação com o impacto que o aumento no número de pessoas tem - e terá - no planejamento urbano das metrópoles. Em projeto de pesquisa realizada por Dimitris Kyritsis, engenheiro de Topografia e Geomática da TU Delft, na Holanda, oito gateways (pontos de ligação, como roteadores) foram colocados nas porções transversais de diversas ruas da cidade de Dordrecht a fim de monitorar pedestres, ciclistas e veículos (LIBELIUM, 2017). Com o objetivo geral de detectar os padrões de ocupação e modalidade de transportes no local, a pesquisa buscava melhorar o planejamento urbano no mesmo. Como resultado, fez-se possível a detecção das rotas mais regulares; do volume de carros, bicicletas ou pedestres; e das ruas mais lotadas de acordo com horários específicos, como, por exemplo, a relação da lotação com o horário de funcionamento das lojas (DUYNSTEE et al., 2016).

No contexto de iniciativas no âmbito do varejo, é válido ressaltar o quanto a experiência do usuário com loT difere da experiência sem a aplicação dispositivos conectados. $\mathrm{O}$ ato de ir a uma loja física, por exemplo, trata-se de um evento analógico, limitado ao horário comercial, em que, no meio, não há troca nem armazenamento de dados entre loja e cliente - pelo menos não além de uma possível transação do cartão de crédito. Nesse cenário, o cliente passa de uma arara a outra; vai ao provador; e, ou realiza a compra, ou simplesmente desiste da mesma e vai para casa. Com o advento da compras on-line no contexto do comércio eletrônico, o ato de compra anexa uma trilha de dados para cada fase do processo. Tem-se, portanto:

- Monitoramento de busca, de rolagem (scroll de tela), de cliques e toques de tela, heatmaps, entre outros;

- Recomendações de produtos e descontos;

- Ratings e demais avaliações do usuário em relação ao serviço prestado e produto;

- Pagamento eletrônico; 
- Integração de perfis de clientes em sistemas que medem e otimizam o processo de comprar.

Por fim, a Internet das Coisas introduz o conceito de Smart Retail (ou Varejo Inteligente), que busca unificar os dados, as interações loja-cliente-produto e o mundo físico. Como explicam Di Rienzo et al. (2016), a criação de serviços inovadores para varejo se vale do fato que este vem sofrendo com a concorrência e o crescimento do comércio eletrônico e das grandes redes de shoppings e supermercados. Assim, a ideia da loT é a de que a interação (o fazer compras), juntamente com os dados, transcenda o computador e o dispositivo móvel do cenário do comércio eletrônico, e se incorpore literalmente a qualquer objeto ou infraestrutura de uma loja física. No exemplo de interações in-stores (dentro de lojas físicas) que geram dados simultaneamente à entrada do cliente na loja, têm-se:

- Check-in do cliente pelo dispositivo móvel;

- Conexão do cliente ao Wi-Fi ou por meio de um beacon;

- Escaneamento de uma etiqueta RFID ou de qualquer outro sensor que indique o interesse do cliente em um objeto ou promoção;

- Provador de roupas com o auxílio de um Smart Mirror (Espelho Inteligente), no qual se poderia procurar por vários tamanhos, estampas e cores de peças de roupas e de acessórios, além de permitir a sobreposição digital de produtos sobre a imagem do cliente através de realidade aumentada - o FX Mirror (Figura 1), por exemplo, promete medições automáticas e precisas do corpo da cliente, compartilhamento instantâneo de imagens nas redes sociais, e apresentação 3D em tempo real das medidas corporais da cliente com imagens sobrepostas de roupas e acessórios.

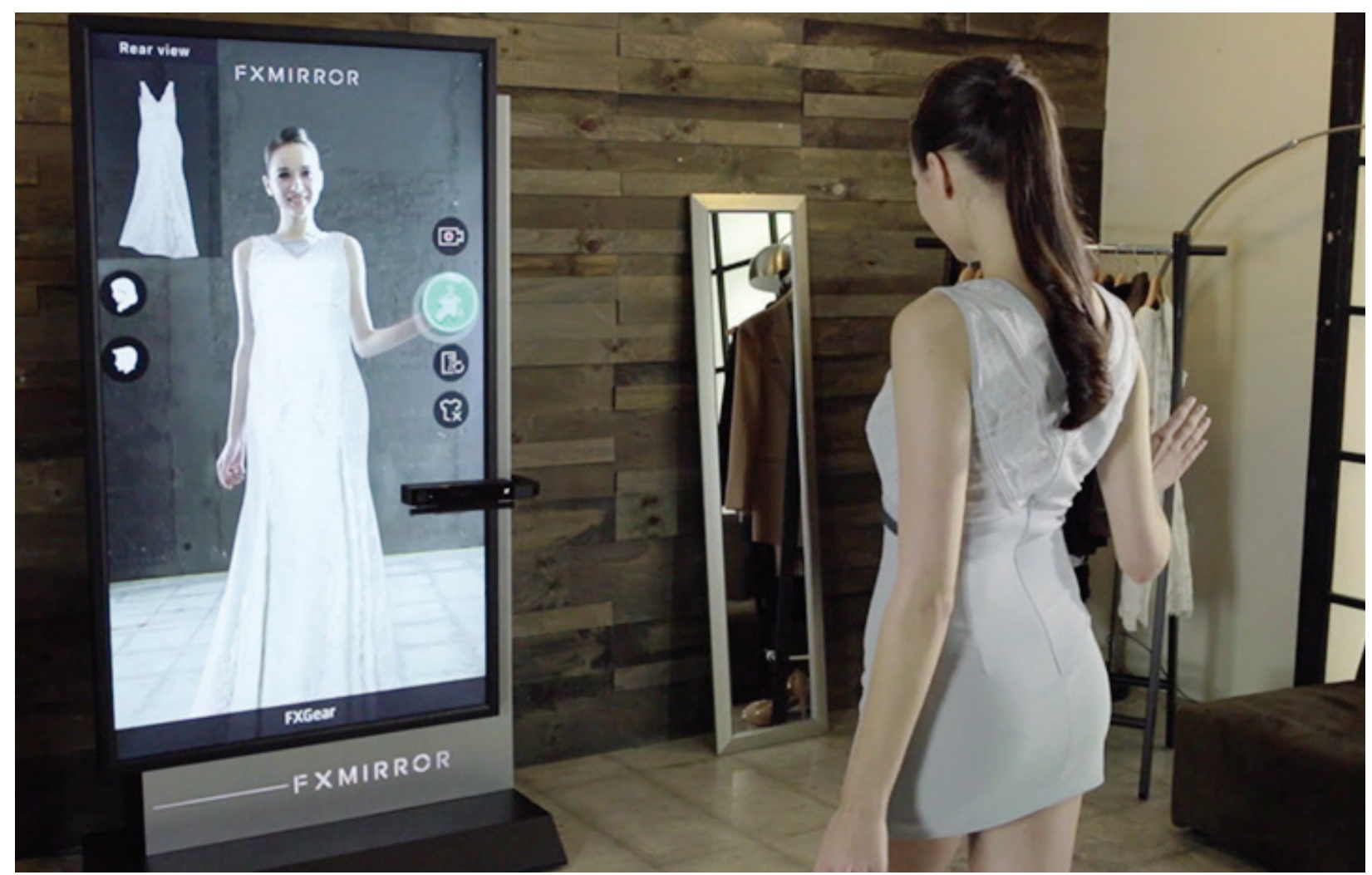

Figura 1 - Cliente utilizando a ferramenta do FX Mirror de sobreposição digital de roupas. Fonte: www.fxmirror.net (2018) 


\subsection{Interfaces: inputs e outputs}

Em um mundo em que os dados são gerados por nossas interações, a interface torna-se a parte tangível de um todo muito mais profundo e intangível (GROOPMAN, 2016). O que é visível, tangível e perceptível é a interface apresentada ao usuário da IoT, à medida que ele a experimenta através dos sentidos. De acordo com Rowland et al. (2015), há uma variedade de maneiras pelas quais usuários podem fornecer inputs (entradas) (Quadro 2) e receber outputs (saídas) (Quadro 3) de um produto ou serviço, que vão dos controles físicos mais tradicionais, com as telas sensíveis ao toque presentes nos smartphones, aos de reconhecimento de voz e gestos até a detecção de sensações térmicas do corpo humano.

\begin{tabular}{cc}
\hline Inputs de & Usados em \\
\hline Toque & Controles físicos, telas sensiveis ao toque \\
\hline Pressão & Interfaces tangíveis \\
\hline Fala & Reconhecimento de voz \\
\hline Corpo (todo) & Reconhecimento de gestos, sensor de proximidade \\
\hline Monitoramento da resistência galvânica da pele* & Deteç̧ão de estresse \\
\hline Pensamentos & Interfaces Cérebro-Computador \\
\hline Batimentos cardíacos & Detecção de estresse, ansiedade, sono etc. \\
\hline * Mede a condutância elétrica da pele, ou seja, a atividade elétrica das glândulas que produzem suor nas \\
palmas das mãos e pontas dos dedos, mais sensíveis às emoções e pensamentos.
\end{tabular}

Quadro 2 - Relação dos modos de input de dados e de ações do usuário e para que podem ser usados.

Fonte: adaptado de Rowland et al. (2015)

\begin{tabular}{cc}
\hline Outputs de & Usados em \\
\hline Audição & Áudio e saida de voz \\
\hline Sensação tátil & Vibrações, formas e feedback de força \\
\hline Cheiro & Aromas \\
\hline Sensação térmica & Temperatura \\
\hline
\end{tabular}

Quadro 3 - Relação dos modos de output e para que podem ser usados. Fonte: adaptado de Rowland et al. (2015)

Ainda segundo Rowland et al. (2015), apesar das diversas possibilidades de inputs e outputs, o design de interfaces e de interação para produtos conectados da loT ainda se caracteriza pelas interfaces web/mobile de click-and-point (clicar e apontar, do cursor do mouse) e de tela sensível ao toque. Groopman (2016, tradução nossa) reitera ao afirmar que a cultura hoje (do ponto de vista ocidental) é "viciada em 
telas", porém, deve-se pensar para além dos limites da tela. Isto, posto que, interfaces em tela são menos propensas a serem usados em dispositivos embutidos da loT. De fato, o aumento no uso de smartphones nos últimos anos estabeleceu um novo precedente de mobilidade para a forma como se pensa em interagir com a internet. Mas será que ações como swiping, pinching e spreading (Figura 2), ou mesmo digitar em pequenos teclados, são realmente as únicas experiências de usuário conectado?
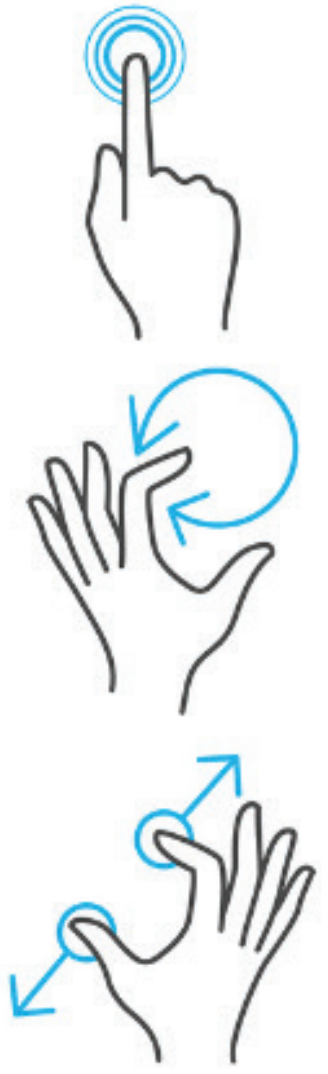
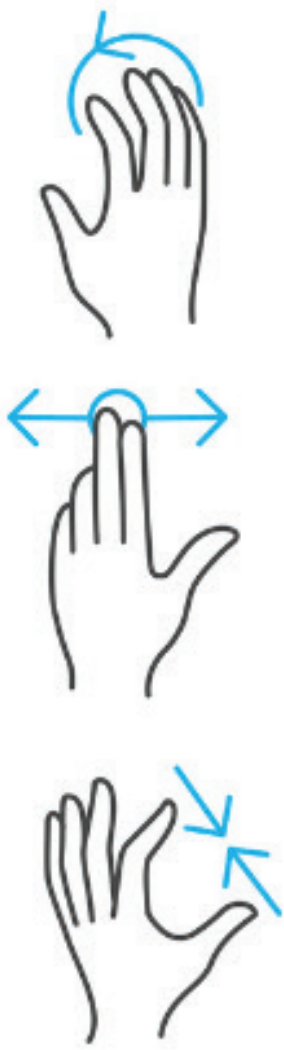
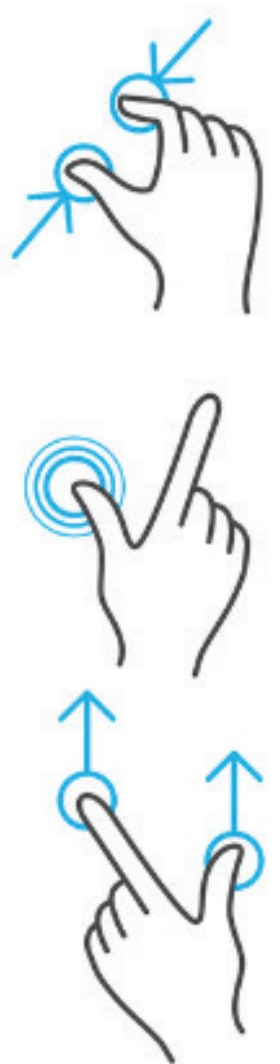
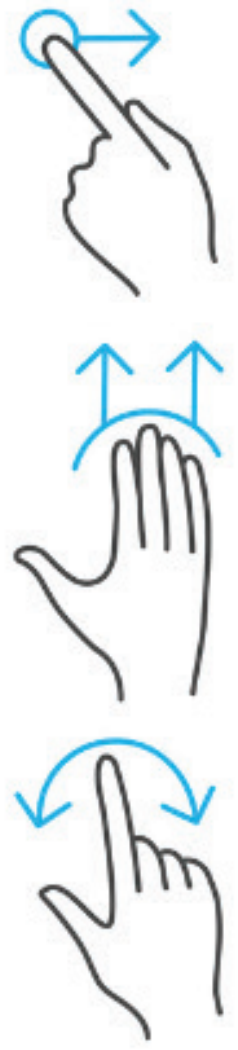

Figura 2 - Exemplo de gestos para interfaces em tela sensível ao toque. Fonte: vectorstock.com (2018)

A Internet das Coisas oferece uma oportunidade para redefinir a forma como se interage com dispositivos digitais. Para Victor (2011), há diversas e intricadas maneiras de se interagir utilizando as mãos no dia a dia, e o uso de interfaces em telas sensíveis ao toque estaria, de certa forma, atrapalhando essas interações cotidianas. Horan (2016) acrescenta que o caráter visual, ainda que impactante, não é o único modo de ter experiências com tecnologias, sendo que outros modos estão ganhando força e, por vezes, desbancado as interfaces visuais como um todo. Exemplos à tal afirmação podem ser visto em iniciativas como as a seguir.

\subsubsection{Exemplo de interface de áudio e saída de luz}

Como exemplo tem-se o frasco de pílulas falante como interface não visual, da AdhereTech $^{7}$ (Figura 3), um frasco de medicamentos que faz parte de um serviço co-

$7 \quad$ AdhereTech: <https://www.adheretech.com/>. Acesso em: 22 dez. 2017. 
-nectado, ou seja, uma plataforma para otimização de adesão médica. No ato da compra de medicamentos, os pacientes retiram o frasco pré-configurado com o farmacêutico e o devolvem para reutilização/reciclagem quando a receita se esgota. O frasco é equipado para fornecer lembretes visuais e de áudio, e simultaneamente, alertar profissionais médicos quando os pacientes não agem de acordo com esses lembretes. Além disso, os pacientes podem selecionar a mídia em que preferem receber alertas - seja pelo smartphone, ou diretamente pelo frasco. O produto combina interfaces de hardware e software criadas para usuários de perfis múltiplos: pacientes, médicos, enfermeiros, farmacêuticos, pesquisadores e assim por diante.

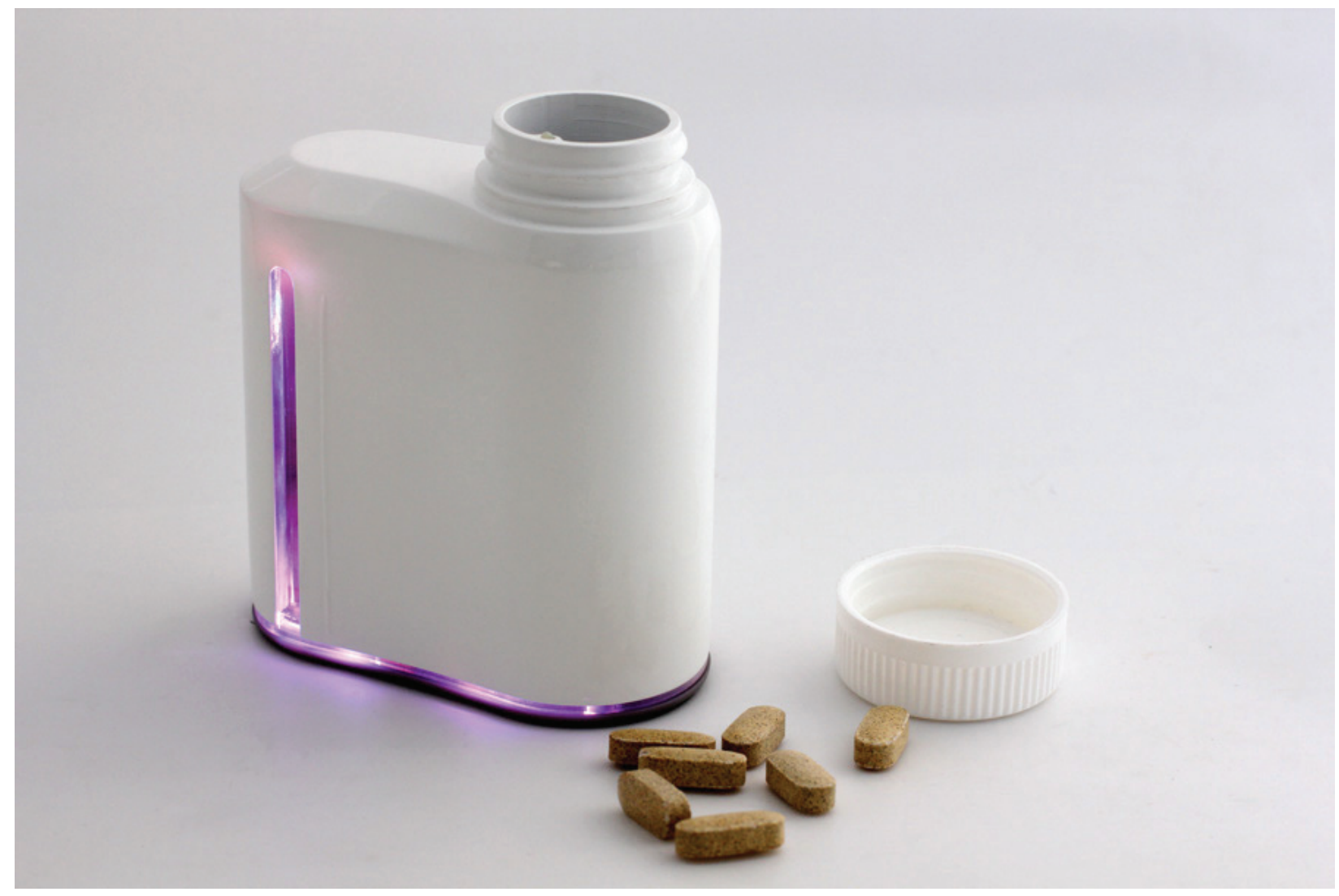

Figura 3 - Frasco de medicamentos equipado para fornecer lembretes visuais e sonoros. Fonte: www.adheretech.com (2018)

\subsubsection{Exemplo de interface gestual e de toque}

O pequeno dispositivo sem fio da $\mathrm{Knocki}^{8}$ (Figura 4) promete converter qualquer superfície comum em uma interface de controle, um exemplo de interface gestual e de toque. O dispositivo desencadeia a ação apropriada com base em quantas vezes um usuário toca na superfície (quando alguém bate na porta, por exemplo). Os usuários podem personalizar (via aplicativo móvel) quais ações específicas são acionadas pelo número de batidas. Através de integrações com uma variedade de dispositivos conectados, bem como serviços de software, os usuários podem programar pedidos complexos, como bater duas vezes para empurrar automaticamente o sono no alar- 
-rme e iniciar a cafeteira; ou simples, como bater três vezes para localizar um smartphone perdido.

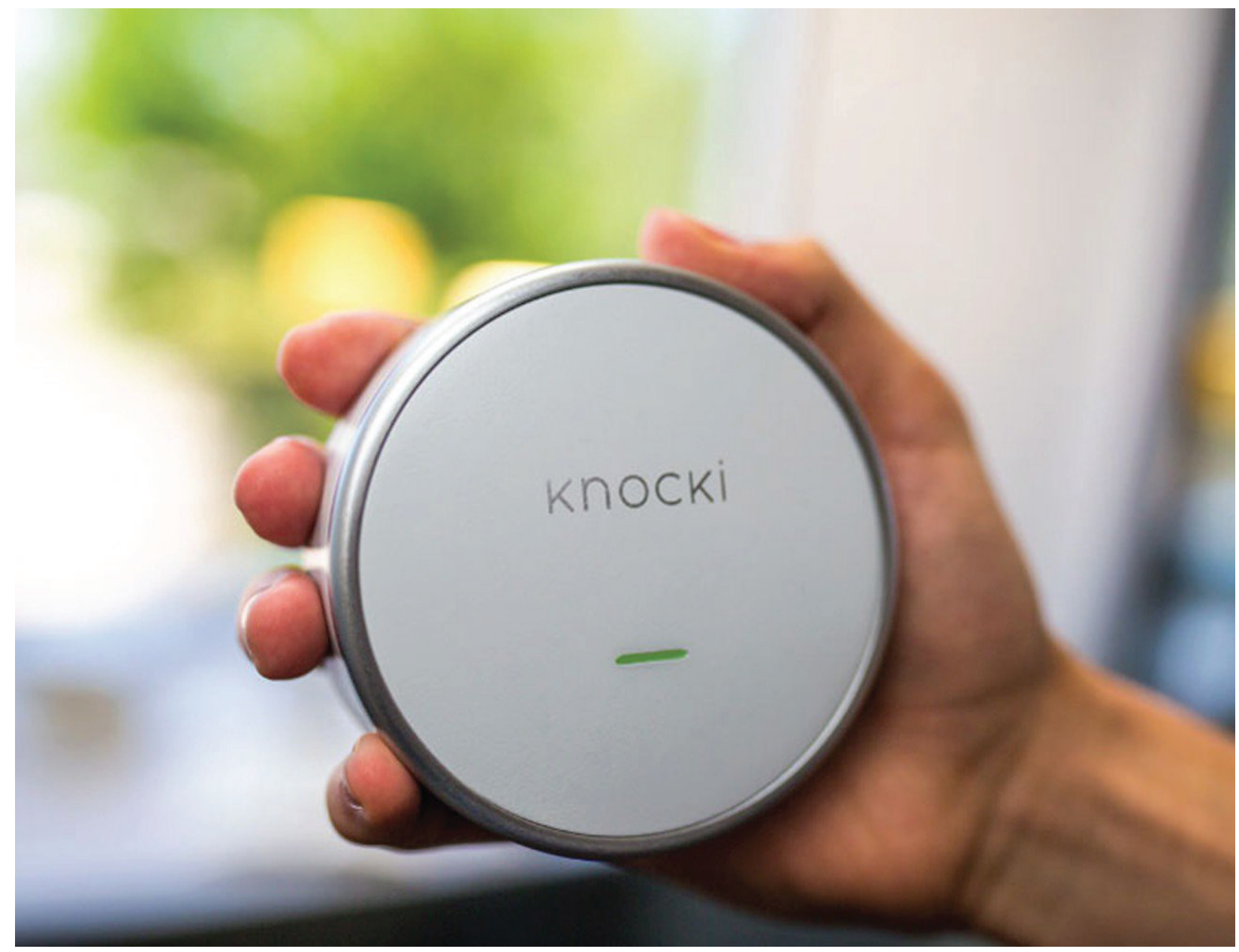

Figura 4 - 0 dispositivo sem fio da Knocki promete converter qualquer superfície comum em uma interface de controle. Fonte: knocki.com (2018)

\subsubsection{Exemplo de interface de áudio, voz e gestos}

Uma assistência digital na orelha como forma de interface de áudio, voz e gestos, da Sony Xperia Ear9 (Figura 5), faz com que fones de ouvido - que se parecem com qualquer outro fone de ouvido sem fio - falem silenciosamente nos ouvidos dos usuários, ajudando na navegação, pesquisa, música, informações climáticas, agendamento e envio e recebimento de mensagens. Os dispositivos da Sony também estão equipados com acelerômetros e giroscópios, e podem detectar gestos de confirmação de um comando. Os usuários personalizam configurações para o dispositivo em seu aplicativo móvel, incluindo notificações, integrações e outras preferências de som.

$9 \quad$ Sony Xperia Ear: <https://www.sonymobile.com/>. Acesso em: 12 dez. 2017. 


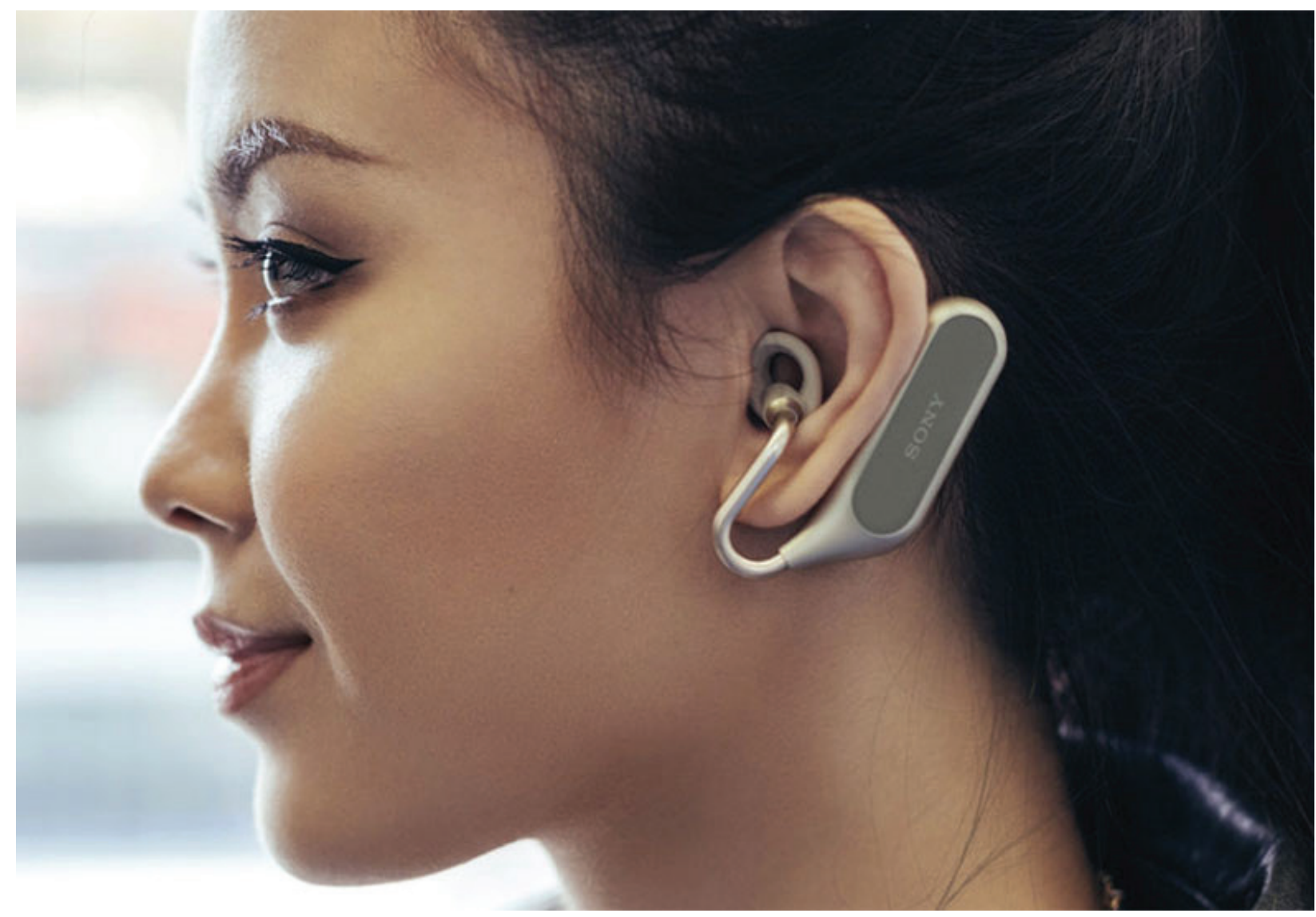

Figura 5 - 0 assistente pessoal da Sony, na forma de fones de ouvido. Fonte: www.sonymobile.com (2018)

\section{Conclusão}

Neste artigo, discutiu-se como a evolução dos dispositivos computacionais, até o entendimento da existência de um ecossistema da Internet das Coisas (IoT), transformou o modo como seres humanos interagem com tecnologias. No contexto industrial, sob a alcunha de IloT, o uso de dispositivos conectados abrange muitos casos de uso e modelos de aplicação. Inicialmente focada apenas na otimização da eficiência operacional e racionalização, automação e manutenção de recursos, hoje, a expectativa é a de que a convergência de loT, inteligência artificial e big data (coleta imensa de dados) introduza as tecnologias da próxima geração, incluindo as Interfaces Cérebro-Computador. Enquanto na IloT o foco está intrinsecamente nas aplicações, no âmbito da CloT, voltado aos consumidores, o objetivo se relaciona mais às novas e imersivas experiências centradas no usuário final.

Fato é que, algumas mudanças proporcionadas pela Internet das Coisas são apenas incrementais, enquanto outras tem o potencial de serem realmente transformadoras. Em conclusão, um benefício fundamental da loT está na capacidade de aproveitar uma coleta imensa de dados e transformá-los em ações para indústria e consumidores, de maneiras anteriormente não vistas. Parte-se aqui da noção de que, para além de definir o que é a Internet das Coisas simplesmente descrevendo todas as características do ecossistema, faz-se fundamental refletir quanto ao propósito da IOT. Nessa perspectiva, os dados são uma parte crucial, embora sozinhos não sejam 
suficientes. O aspecto da interconectividade e da integração de dispositivos, somado a essa coleta de dados, são o que apontam para uma realidade em que dispositivos, pessoas, processos e informações estão mais interligados do que nunca.

\section{Referências}

ASHTON, K. That 'Internet of Things' Thing. RFID Journal, v. 22, n. 7, 2011.

BASSI, A.; HORN, G. Internet of Things in 2020: A Roadmap for the Future. European Commission: Information Society and Media, v. 22, p. 97-114, 2008.

CAMPBELL-KELLY, M.; ASPRAY, W.; ENSMENGER, N.; YOST, J. R. Computer: A History of the Information Machine. 3a Edição. Westview Press, 2014.

CASTELLS, M. The Rise of the Network Society: The Information Age: Economy, Society, and Culture. John Wiley \& Sons, 2011.

COMPTIA. 2015. In: CompTIA, 2005. (http://www.comptia.org/resources/ internet-of-things-insights-and-opportunities)

DI RIENZO, A.; TAGLIAFERRI, P.; ARENELLA, F.; GARZOTTO, F.; FRÀ, C.; CREMONESI, P.; VALLA, M. Bridging Physical Space and Digital Landscape to Drive Retail Innovation. In: Proceedings of the International Working Conference on Advanced Visual Interfaces. ACM, p. 356-357, 2016.

DUYNSTEE, C. A. N. L.; HAAYEN, M. J.; KYRITSIS, D.; ORTEGA-CORDOVA, L. M.; SAMAT, S. N. N. Smart City Dordrecht: Identification of Pedestrian Movement Patterns with Wi-Fi Tracking Sensors. Geomatics Synthesis Group Project Report, 2016.

EASTERLING, K. An Internet of Things. E-flux Journal, v. 31, 2012.

GĄSIOREK, A. The Complete Beacon Industry Report: Current Technology and Recent History. In: Kontakt.io, 2014. (https://kontakt.io/blog/infographic-beacons/)

GERSHENFELD, N. When Things Start to Think. Macmillan, 1999.

GROOPMAN, J. Product Manufacturers: It's Time to Rethink the loT User Interface. TechTarget, 2016. (http://internetofthingsagenda.techtarget.com/feature/Product-manufacturers-Its-time-to-rethink-the-loT-user-interface)

HEUVELDOP, N. Ericsson Mobility Report. Ericsson AB, Technology \& Emerging Business, Estocolmo, Suécia, Tech. Rep., EAB-17, v. 5964, 2017.

HORAN, B. Branded Interactions: Trends and Strategies for Digital-Physical Products. In: Appliance Design Magazine, 2016. (https://www.appliancedesign.com/articles/ 94870-branded-interactions-trends-and-strategies-for-digital-physical-products) 
IBGE. Pesquisa Nacional por Amostra de Domicílios [Internet]. 2014.

KAY, R. Endpoints vs. The Internet of Things. In: Forbes, 2014. (https://www. forbes.com/sites/rogerkay/2014/01/21/endpoints-vs-the-internet-of-things/).

KUNIAVSKY, M. When the Cloud Decides: Designing for Predictive Machine Learning for the IoT. In: O'Reilly Design Conference, jan. 2016. (Comunicação oral). (https://www.scribd.com/doc/296215323/When-the-cloud-decides-Designing-for-predictive-machine-learning-for-the-IoT-O-Reilly-Design-2016)

LELLA, A.; LIPSMAN, A. The US Mobile App Report. Tech. Rep., v. 8, 2014.

LIBELIUM. Detecting Road Modality and Occupancy Patterns to Enhance Urban Planning in Dordrecht Smart City. In: Case Studies, 2017. (http://www.libelium. com/detecting-road-modality-and-occupancy-patterns-to-enhance-urban-planning-in-dordrecht-smart-city)

LUCERO, S. IOT Platforms: Enabling the Internet of Things. In: HIS Technology, 2016. (https://cdn.ihs.com/www/pdf/enabling-IOT.pdf)

MCLUHAN, M. Understanding Media: The Extensions of Man. MIT Press, 1994.

MIRANDA, J.; MÄKITALO, N.; GARCIA-ALONSO, J.; BERROCAL, J.; MIKKONEN, T.; CANAL, C.; MURILLO, J. M. From the Internet of Things to the Internet of People. IEEE Internet Computing, v. 19, n. 2, p. 40-47, 2015.

MOON, B. Internet of Things \& Hardware Industry Overview 2016. SparkLabs Global Ventures, 2016.

REVELL, S. Internet of Things (IOT) and Machine to Machine Communications (M2M) Challenges and Opportunities. Final Paper, London, UK Google Scholar, 2013.

ROMKEY, J. Toast of the IOT: The 1990 Interop Internet Toaster. IEEE Consumer Electronics Magazine, v. 6, n. 1, p. 116-119, 2017.

ROWLAND, C.; GOODMAN, E.; CHARLIER, M.; LIGHT, A.; LUI, A. Designing Connected Products: UX for the Consumer Internet of Things. O'Reilly Media, Inc., 2015.

TESLA, N. When Woman is Boss. Colliers, Edição de 30 de Janeiro, 1926. In: An interview with Nikola Tesla by John B. Kennedy. 21st Century Books, 2013. (http:// www.rawscience.tv/when-woman-is-boss-tesla-on-wifi-and-gender-equality/)

TURING, A. M. Computing Machinery and Intelligence. Mind, v. 59, n. 236, p. 433, Oxford University Press, 1950. 
UNITED NATIONS. World Urbanization Prospects: The 2014 Revision, Highlights. Department of Economic and Social Affairs. Population Division, United Nations, 2014.

VICTOR, B. A Brief Rant on the Future of Interaction Design. Worry Dream. com, 2011.

WEIS, S. A. RFID (Radio Frequency Identification): Principles and Applications. System, v. 2, n. 3, 2007. 